Journal für Mobilität und Verkehr

ISSN 2628-4154

www.dvwg.de

\title{
Methodik einer Machbarkeitsstudie zum Einsatz batterieelektrischer Triebwagen (BEMU) am Beispiel des VVO-Dieselnetzes
}

\author{
Benjamin Ebrecht, Daniel Walter, Ivo Zedlitz, Ulrich Zimmermann* \\ Technische Universität Berlin, Fachgebiet Bahnbetrieb und Infrastruktur, Sekretariat SG 18, Salzufer 17-19, 10587 Berlin, Deutschland
}

\begin{abstract}
Der Einsatz von Fahrzeugen mit alternativen Antriebssystemen im Schienenpersonennahverkehr hat in den letzten Jahren unter anderem durch neue technische Entwicklungen starken Auftrieb bekommen. Aufgrund der Charakteristika entsprechender Fahrzeuge haben sich gleichzeitig neue Fragen an die betriebliche Umsetzung ergeben. Am Beispiel einer Machbarkeitsstudie für den Betrieb von Batterietriebzügen im Verkehrsverbund Oberelbe (VVO) sollen die relevanten Fragen veranschaulicht werden.
\end{abstract}

Schlagwörter/Keywords:

Schienenpersonennahverkehr, Alternative Antriebssysteme, Batteriefahrzeuge, Betriebsplanung

\section{Zero-Emission-Betrieb durch den Einsatz alternativer An- triebe}

Es gilt als anerkannt, dass der Schienenverkehr mit seinem potenziellen Vorteil eines Zero-Emission-Betriebs ein entscheidender Baustein der Klima- und Verkehrswende werden kann [1]. Praktisch sind derzeit rund $60 \%$ des deutschen Schienennetzes elektrifiziert, doch auch bei vollständiger Umsetzung der im Bundesverkehrswegeplan (BVWP) vorgesehenen Aus- und Neubaupläne steigt der Elektrifizierungsgrad nicht über $67 \%$ [2].

Demgegenüber stehen die Kennwerte für den Schienenpersonennahverkehr (SPNV): in diesem Marktsegment werden von den etwa 1.000 SPNV-Linien in Deutschland knapp die Hälfte mit Dieselfahrzeugen betrieben, was auf einen großen Anteil befahrener, nichtelektrifizierter Streckenabschnitte schließen lässt. Dementsprechend muss konstatiert werden, dass der SPNV-Betrieb einen Anteil von 64 \% [3] am Dieselverbrauch des deutschen Schienenverkehrs hat - inklusive entsprechender Auswirkungen auf den $\mathrm{CO}_{2}$-Ausstoß. Die im Rahmen des Forschungsbereiches alternative Antriebssysteme am Fachgebiet erhobenen Daten zeigen, dass diese Linien einen hohen Grad an Diversität aufweisen. Es gibt eine große Anzahl kurzer Linien, die eine Länge von $50 \mathrm{~km}$ nicht überschreiten und sehr ähnliche Charakteristika in Bezug auf den Elektrifizierungsgrad besitzen. Trotzdem ist eine detaillierte Einzelfallbetrachtung geboten, insbesondere wenn man sich in Liniennetzen bewegt und die Interdependenzen zwischen den Linien betrachtet.

Dies bedeutet unter Berücksichtigung der mittelfristig nicht abzusehenden Vollelektrifizierung, dass eine Substitution der Dieselfahrzeuge im SPNV auf anderem Wege erfolgen muss, um das erklärte Ziel eines klimaneutralen Verkehrs bis zum Jahr 2050 zu erreichen [4].

\section{Batterieelektrische Züge}

Aus technischer Sicht zeigen Fahrzeuge mit batterieelektrischem Antrieb das Potential, emissionsfrei bezüglich des Ausstoßes von Schadstoffen und Treibhausgasen zu fahren: In diesem Sinne lokal emissionsfrei fahren diese in jedem Fall, bei Nutzung von Strom aus regenerativen Energiequellen ist der Betrieb tatsächlich auch auf nichtelektrifizierten Streckenabschnitten nahezu klimaneutral. Nachdem Fahrzeuge mit Blei-Säure-Akkus für knapp 100 Jahre bis 1995 in Deutschland unterwegs waren, ist diese Fahrzeugform aktuell nicht in Deutschland im Einsatz [5]. Jedoch gibt es eine Renaissance dieses Fahrzeugtyps auf Basis der aktuellen Lithium-lonen-Technologie: Bereits im März 2014 ging ein Akku-Hybridtriebzug in Japan in Betrieb [6], auf der InnoTrans 2018 stellten die Hersteller Bombardier, Siemens und

\footnotetext{
* Korrespondierender Autor.

E-Mail: uzimmermann@railways.tu-berlin.de (U. Zimmermann)
} 
Stadler jeweils eigene Prototypen vor [7] [8] [9]. In Deutschland wurde im Sommer 2019 eine erste Fahrzeugserie für den kombinierten Akku-/Oberleitungsbetrieb bestellt [10]. Trotz der begrenzten Reichweite von Akkufahrzeugen lassen sich grundsätzlich zahlreiche SPNV-Linien mit ihnen bedienen: Die Länge der nichtelektrifizierten Abschnitte beträgt in etwa jedem zweiten Fall weniger als $40 \mathrm{~km}$ - wo mindestens eine Teilelektrifizierung vorhanden ist, können die Akkus im Oberleitungsbetrieb nachgeladen werden [2]. Fehlt der Zugang zur Oberleitung auf der gesamten Linienlänge, ist zu untersuchen, ob die Errichtung von Ladeinfrastruktur zielführend ist. Es ist dabei sinnvoll, die konkrete Eignung von Batterietriebzügen (engl. BEMU - Battery Electric Multiple Unit) für den Einsatz in bestimmten Liniennetzen zu untersuchen.

\section{Machbarkeitsstudie für den Verkehrsverbund Oberelbe}

Der Verkehrsverbund Oberelbe (VVO) hat die TU Berlin mit einer Untersuchung seines Dieselnetzes beauftragt, deren Ergebnis eine Machbarkeitsstudie für verschiedene Szenarien unter unterschiedlichen Bedingungen für den Einsatz von Batterietriebzügen ist. Als Kernfragen waren zu beantworten:

- Können die aktuell gefahrenen Fahrpläne auch mit den untersuchten Fahrzeugen absolviert werden?

- An welchen Stellen sind eventuell zusätzliche Infrastrukturmaßnahmen in Form von Lademöglichkeiten notwendig?

- Wie stellt sich die Wirtschaftlichkeit im Vergleich zu anderen Fahrzeugkonzepten dar, im konkreten Fall zum Dieselbetrieb?

- Welche Ausschreibungsmodelle bieten sich für diesen neuen Typ von Fahrzeugen an?

Die Vorgehensweise zeigt sich ergebnisgetrieben-strukturiert: Es wurden zuerst die einzelnen Linien detailliert analysiert und vorhandene Infrastruktur zur Nachladung der Fahrzeuge identifiziert. Aufbauend auf den erhobenen Daten erfolgte eine Simulation des Betriebes mit Batteriefahrzeugen. Wenn die Lademöglichkeiten im Status Quo nicht ausreichend sind, wurden pro Linie mehrere Szenarien an Infrastrukturmaßnahmen entworfen und anschließend eine Vorzugsvariante ausgewählt. Am Ende steht für jede Linie die Aussage, ob diese im Status Quo befahrbar ist und wenn nicht, welche Maßnahmen geeignet sind, um dies zu ändern.

Ziel des Artikels soll in diesem Fall allerdings nicht eine Vorstellung der Ergebnisse der Machbarkeitsstudie sein, sondern vielmehr eine Darstellung der Untersuchungsmethodik. Sofern Ergebnisse der Studie dabei verwendet werden, dient dies nur dazu, die Problemstellung verständlicher darzustellen. Die Punkte Wirtschaftlichkeit und Ausschreibungsnetze werden in diesem Beitrag nicht behandelt.

\section{Analyse der Linien}

Es wurden fünf Linien im Großraum Dresden analysiert, von denen vier im regulären Taktverkehr bedient werden, die fünfte verkehrt nur für den Ausflugsverkehr an Wochenenden. Abbildung 2 zeigt die Aufteilung der Linienlängen auf elektrifizierte und nicht elektrifizierte Abschnitte. Dabei ist hervorzuheben, dass derzeit zwei Linien keinen Zugang zur Oberleitung haben und BEMU-Fahrzeuge während der Umläufe nicht geladen werden könnten. Inwieweit die Elektrifizierung der Gleise des Bahnhofs Heidenau ausreicht, muss noch endgültig geklärt werden. Sie werden bis dahin als nichtelektrifiziert betrachtet. Weiterhin müssen teils erhebliche Höhenunterschiede überwunden werden, im Fall der Linie RB 71 sind dies 300 m und für die Linie RB 72630 m. Zudem weist insbesondere die Trassierung durch das Müglitztal (RB 72) viele enge Bögen auf, die Einfluss auf den Energieverbrauch der Fahrzeuge haben.

Wie in Abbildung 1 zu sehen, umfasst das untersuchte Liniennetz mehrere Strecken im Großraum Dresden, elektrifiziert sind diese nur dort, wo sie den grün hervorgehobenen Abschnitt Heidenau - Dresden Hbf - Dresden-Neustadt - Dresden-Klotzsche befahren. Dabei ist zu beachten, dass sowohl in Heidenau als auch in Dresden-Klotzsche nicht alle Bahnsteiggleise elektrifiziert sind.

Auf den Linien RB 33, RB 34 und RB 72 wird aktuell zu den meisten Zeiten im Stundentakt gefahren, zum Teil gibt es zusätzliche Verstärker und Ausdünnungen am Wochenende auf einen Zweistundentakt (vgl. Abbildung 2). Die Linie RB 71 verkehrt von Pirna bis Neustadt (Sachsen) im Stundentakt, von dort bis Sebnitz meist nur im Zweistundentakt. Der RE 19 bietet als Ski- und Wanderzug eine direkte Fahrtmöglichkeit zwischen Dresden und Altenberg.

\section{Simulation des Betriebes mit dem TALENT 3 Batterietrieb- zug}

Die konkreten Fahrdynamik- und Energiebedarfssimulationen wurden auf Basis detaillierter Fahrzeugparameter des Triebzuges TALENT 3 BEMU durchgeführt und beinhalten sowohl den Ladezustand der Batterie über den Fahrtverlauf als auch eine sekundengenaue Fahrzeitrechnung. Hierfür wurden neben den von Bombardier Transportation selbst stammenden Fahrzeugdaten auch detaillierte Infrastrukturgrunddaten von DB Netz zugrunde gelegt. Es wurden alle relevanten Parameter mit Einfluss auf den Energieverbrauch berücksichtigt, sowohl diejenigen aus dem Fahrzeug selbst als auch von der Infrastrukturseite wie beispielsweise Streckenneigung und Kurvenradien. Zusätzlich wurden die Fahrplanvorgaben des VVO berücksichtigt.

Aus dem ersten Simulationsdurchgang ging hervor, dass mehrere Linien im aktuellen Zustand nicht ohne weiteres mit Batterietriebzügen betrieben werden können. Hierzu sei klargestellt, dass dieses Ergebnis und alle Aussagen bezüg- 
Abbildung 1: Lage des VVO-Dieselnetzes mit elektrifizierten Abschnitten

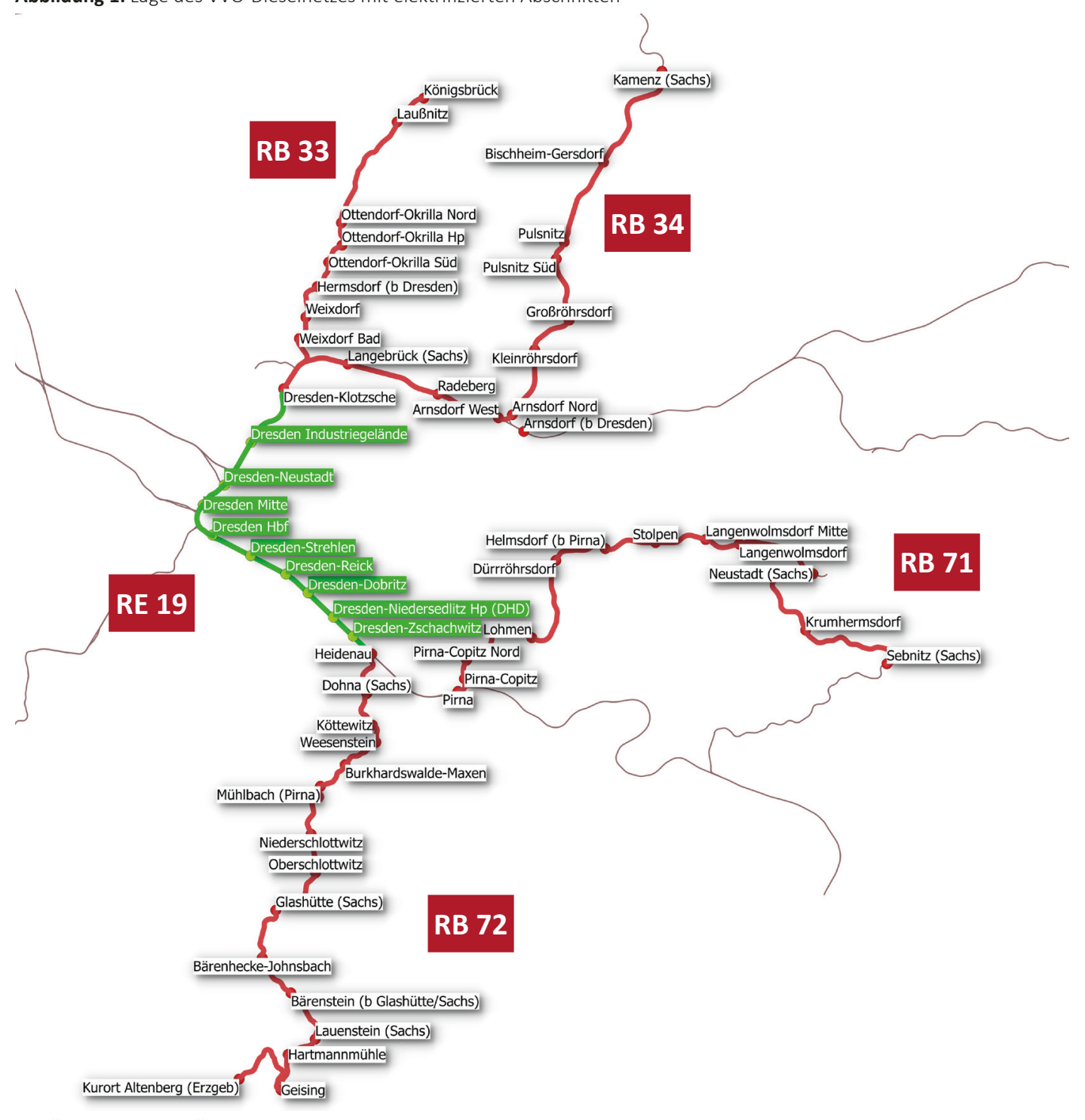

Quelle: Eigene Darstellung

Abbildung 2: Aufteilung der Linien nach elektrifizierten und nicht elektrifizierten Abschnitten

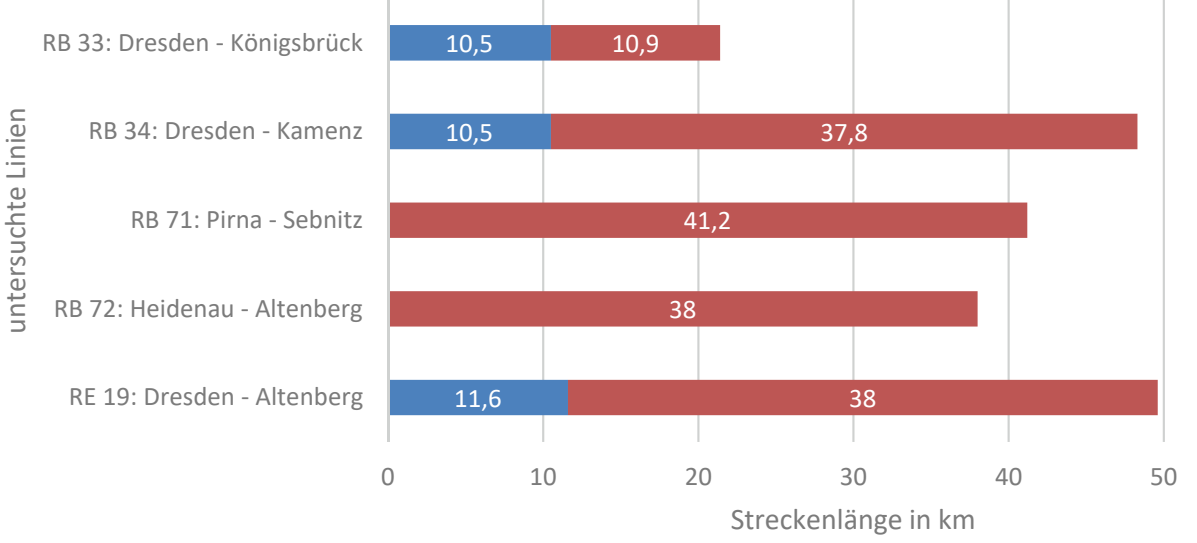

घ elektrifizierte Streckenlänge घ nicht elektrifizierte Streckenlänge

Quelle: Eigene Darstellung 
lich der Eignung von Strecken für den Batteriezugbetrieb im VVO-Netz ausschließlich für das oben beschriebene Fahrzeug mit den vorgegebenen Randbedingungen gilt. Sobald sich im Bereich der Fahrzeugentwicklung Veränderungen beispielsweise der Fahrzeugeigenschaften ergeben, ändern sich damit sofort auch die Simulationsergebnisse. Denkbar sind zum Beispiel Weiterentwicklungen im Bereich der Batterietechnik, die eine deutliche Verbesserung der gravimetrischen Energiedichte ergeben, sodass bei gleichbleibender Masse mehr Energie gespeichert werden kann. Angetrieben durch die Nachfrage nach leistungsfähigen Batteriesystemen ist in den kommenden Jahren von einer deutlichen Steigerung der Energiedichte in Batterien auszugehen [11].

\section{Detaillierte Betrachtung der Energiebedarfs- und Lade- kurve}

Für die Simulation und Darstellung von Energiebedarf und Nachladung im Laufe mehrerer Fahrten auf einer Linie wurde vom Fachgebiet Methoden der Produktentwicklung und Mechatronik der TU Berlin die Software eFlips (Electric Fleet and Infrastructure Planning/Simulation) entwickelt [12]. In Abbildung 3 wird eine solche Visualisierung am Beispiel der Strecke Dresden - Königsbrück dargestellt. In den gelb unterlegten Abschnitten ist eine Oberleitung vorhanden und somit die Möglichkeit zur Nachladung der Batterien gegeben. Der zwischen 0 und $100 \%$ dargestellte Bereich bezeichnet den dauerhaft nutzbaren Bereich der Batterie. Darunter liegt ein Puffer- und Tiefenentladebereich, der für den Einsatz in Störungsfällen genutzt werden kann. Da hiermit aber eine Lebensdauerverkürzung der Batterie einhergehen kann, ist er für den Regelbetrieb nicht einsetzbar. Der Tiefentladebereich ist in der Abbildung aus Platzgründen nicht vollständig dargestellt. Der Ladezustand (SoC = State of Charge) bezieht sich dabei auf die nutzbare Batteriekapazität. Die Konvention, die gesamte Strecke zwei Mal in beide Richtungen zu befahren, resultiert aus folgender Erkenntnis: Auf diese Weise kann gewährleistet werden, dass der Betrieb nicht nach mehreren Umläufen durch eine mit der Zeit immer leerer gewordene Batterie behindert wird, sondern theoretisch unbegrenzt lang mit dem gleichen Ladeintervall fortgeführt werden kann. Eine größere Pause zum Nachladen nach mehreren Umläufen ist nicht vorgesehen.

Abbildung 3: eFlips-Ausgabe am Beispiel Dresden- Königsbrück

KBS: 226,1 von Dresden Hbf tief nach Königsbrück

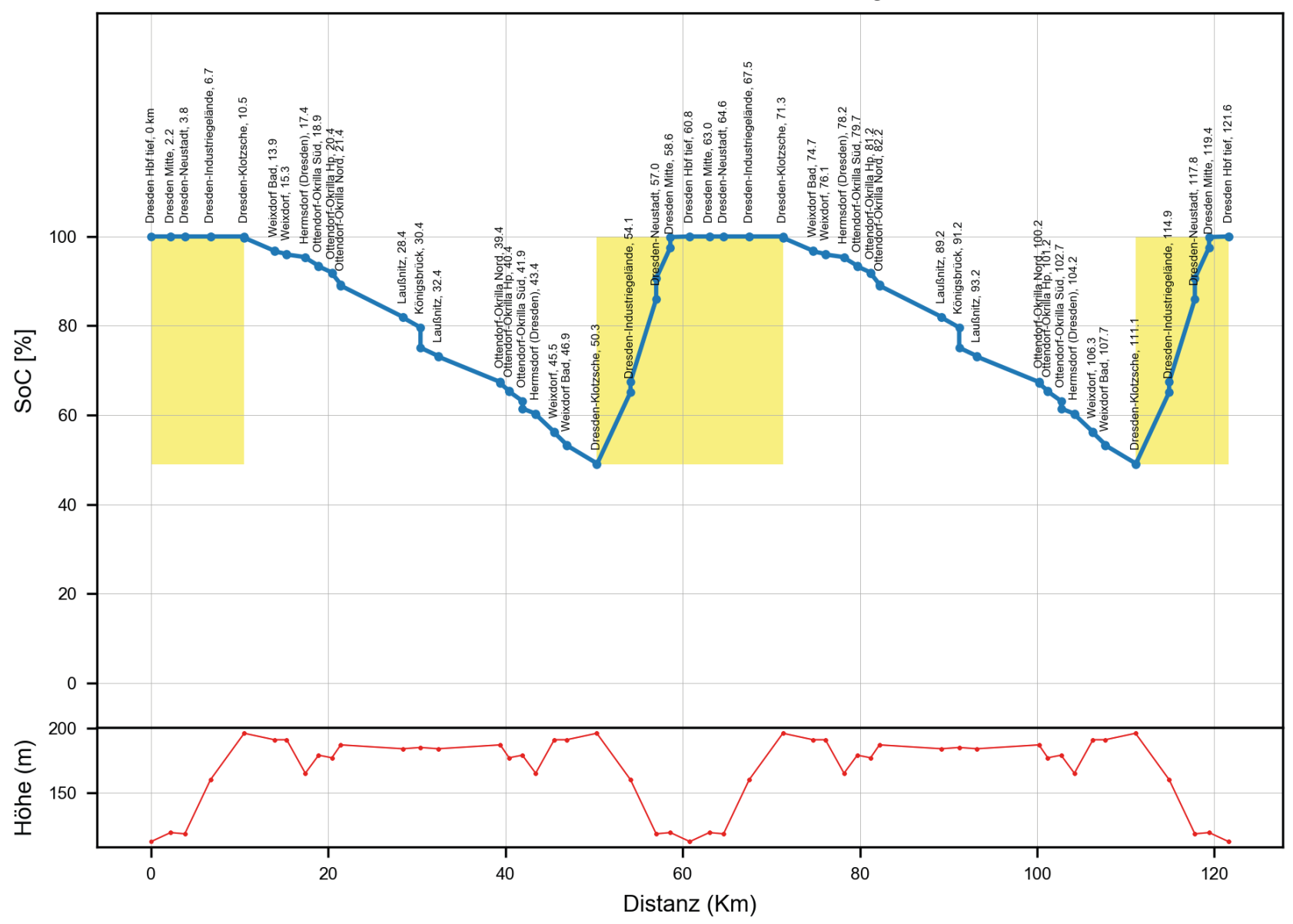

Quelle: MPM 
Anhand des Beispiels der Wende ohne Oberleitung in Königsbrück wird ein Einflussfaktor auf die Befahrbarkeit der Linien deutlich: Auch während der Wende sinkt der Ladestand der Batterie, da die Hilfsbetriebe auch im Stand versorgt werden. Auf der x-Achse wird die Distanz abgetragen, weswegen Lade- und Entladevorgänge im Stand durch eine vertikale Linie des Energieverlaufs dargestellt werden. Eine eventuelle Versorgung der Hilfsbetriebe des Fahrzeugs (z.B. Heizung oder Klimaanlage) über eine externe Steckereinspeisung wurde hier nicht berücksichtigt. Die Auswirkungen auf den Energieverbrauch sind aufgrund der meist kurzen Wendezeiten eher gering. Bei einer langen Wendezeit oder einer Abstellung im nichtelektrifizierten Bereich kann eine solche Einspeisung sinnvoll sein.

Positiv wirkt sich hier auch aus, dass der Abschnitt zwischen Dresden Hbf und Dresden-Klotzsche elektrifiziert ist und die Batterie somit über eine längere Zeit nachgeladen werden kann. Wäre nur der Dresdner Hauptbahnhof elektrifiziert, müsste die Wendezeit dort so lang sein, dass die Batterie für die folgende Fahrt ohne Oberleitung ausreichend geladen wird, was zu Konflikten mit dem aktuellen Fahrplan führen könnte.

\section{Veränderungen durch Errichtung einer Ladestation am Beispiel der RB 34}

Da die Linie RB 33, wie in Abbildung 3 zu sehen ist, bereits ohne Ladeinfrastruktur für Batteriefahrzeuge geeignet ist, wählen wir für eine genauere Betrachtung die RB 34 von Dresden $\mathrm{Hbf}$ über Radeberg und die Arnsdorfer Kurve nach Kamenz aus. Bereits anhand der Kennzahlen ist diese Linie deutlich anspruchsvoller.

Abbildung 4 zeigt die Simulation des BEMU-Einsatzes im Ist-Zustand. Hier ist zu erkennen, dass der Energieverbrauch des nicht elektrifizierten Abschnitts von Dresden-Klotzsche nach Kamenz und zurück (inkl. der Versorgung des Fahrzeuges während der Wendezeit) die Batteriekapazität des Fahrzeuges übersteigt und eine Nutzung des Tiefentladebereiches erfordert. Da dies im Regelbetrieb nicht vorgesehen ist, kann die Linie ohne zusätzliche Infrastruktur nicht mit dem simulierten BEMU-Fahrzeug befahren werden. Auch eine Verkürzung der Wendezeit in Kamenz - sofern sie betrieblich möglich wäre - brächte nur eine marginale Verbesserung, ebenso eine externe Versorgung des Fahrzeugs während der Wendezeit.

Abbildung 4: Simulation der RB 34 im Status Quo

KBS: 227,1 von Kamenz (Sachs) nach Dresden Hbf tief

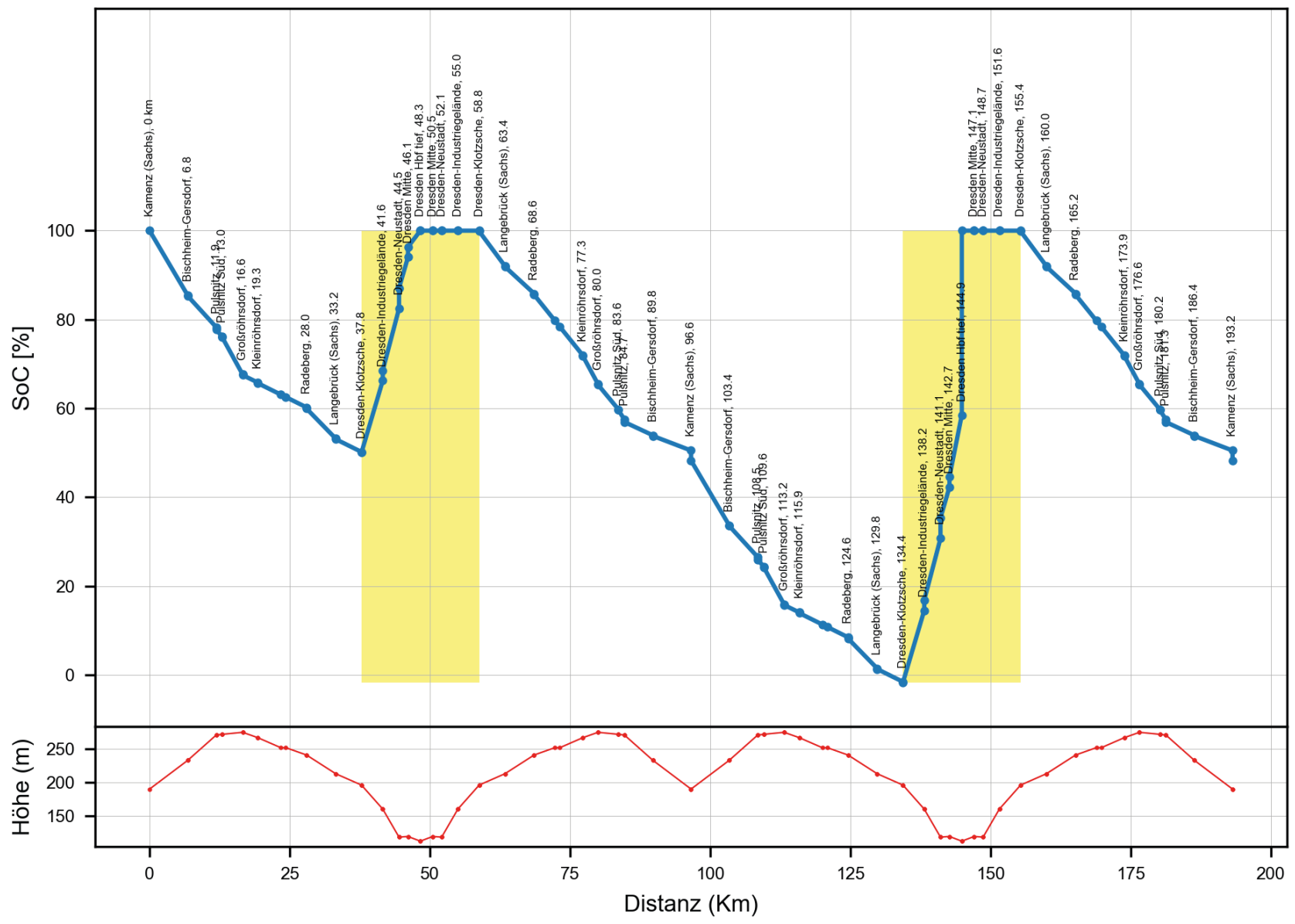


KBS: 227,1 von Kamenz (Sachs) nach Dresden Hbf tief

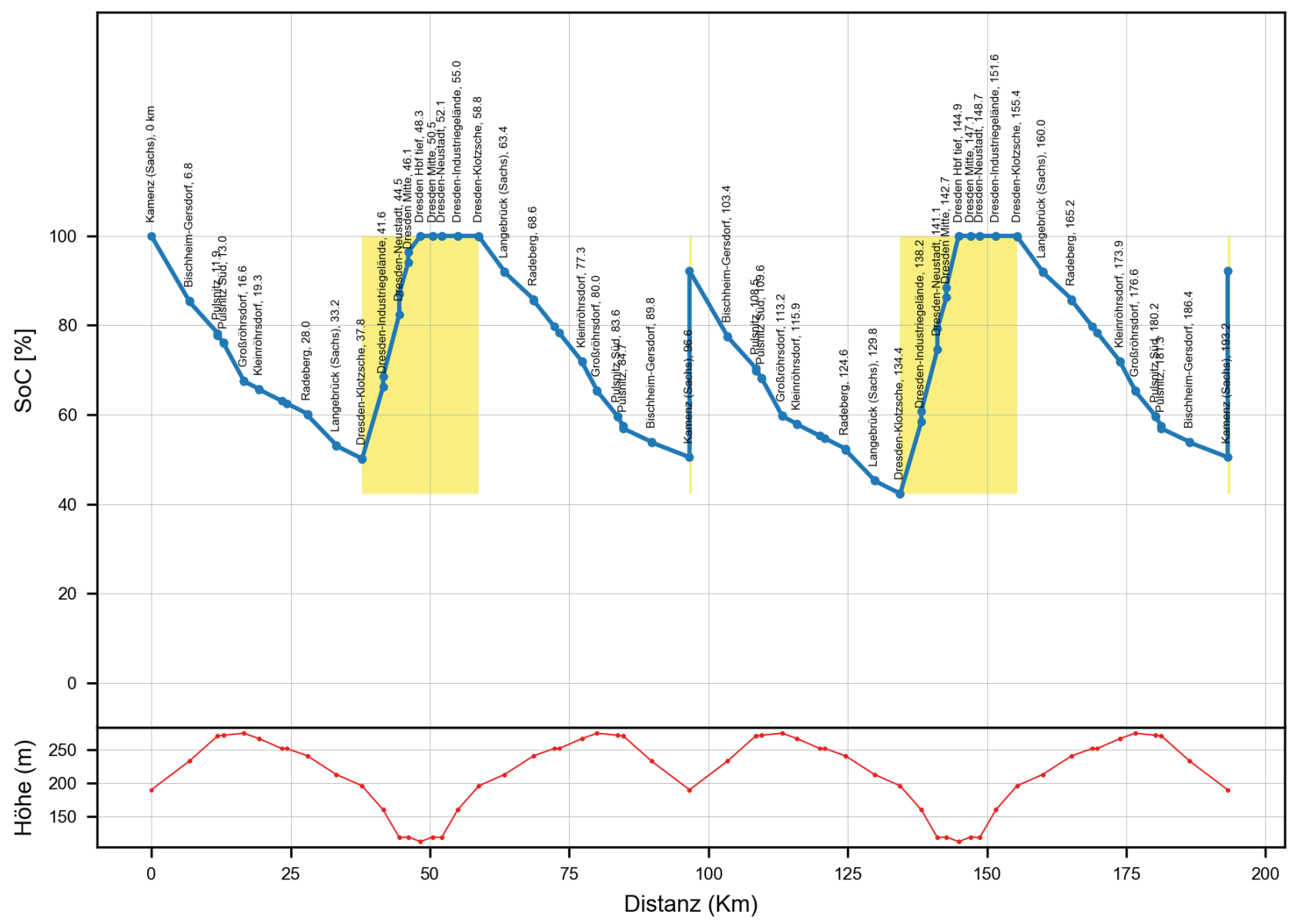

Quelle: MPM

Abbildung 5 hingegen unterstellt die Errichtung einer Ladestation in Kamenz. Der Begriff Ladestation bezeichnet eine Versorgungsinfrastruktur, die eine Ladung der Traktionsbatterie im Stand über den Stromabnehmer ermöglicht. Diese kann über Bahnsteiggleisen positioniert werden, sodass eine Aufladung gleichzeitig mit dem Fahrgastwechsel durchgeführt werden kann. Während der Wendezeiten in Kamenz kann die Batterie dadurch ausreichend wiederaufgeladen werden; anstatt den Tiefentladebereich zu nutzen, sinkt der Ladestand in diesem Szenario nur noch auf etwa die Hälfte des SoC, bevor wieder eine Aufladung erfolgt.

Als weitere Variante wurde die Elektrifizierung von Dresden-Klotzsche bis Arnsdorf unterstellt (siehe Abbildung 6). Diese ist Teil des Elektrifizierungsprojektes Dresden - Görlitz, was bis zum Jahr 2029 abgeschlossen sein soll [13]. Hiervon profitiert die Linie RB 34 bis Arnsdorf bzw. bis vor den Abzweig Arnsdorfer Kurve. Es zeigt sich, dass aus Fahrzeugsicht die Ladestation in Kamenz für die Linie gut geeignet ist, da im Regelbetrieb ein ca. 10 \% kleineres Ladefenster genutzt werden kann.
Im Eckpunktpapier zur Umsetzung der strukturpolitischen Empfehlungen der Kommission „Wachstum, Strukturwandel und Beschäftigung" für ein "Strukturstärkungsgesetz Kohleregionen" des Bundesministeriums für Wirtschaft und Energie ist darüber hinaus die Elektrifizierung der Strecke von Radeberg/Arnsdorf nach Kamenz enthalten [14]. Unter diesen Gesichtspunkten könnten BEMU-Fahrzeuge auf der RB 34 als Brückentechnologie eingesetzt werden, da sie später auch im reinen Oberleitungsbetrieb eingesetzt werden können. Bei Bedarf kann das Batteriesystem entfernt werden, sodass das Fahrzeug dann einem heutigen Elektrotriebwagen entspricht. Die Ladestation in Kamenz wiederum ist so auszulegen, dass sie in eine Vollelektrifizierung der Strecke eingebunden werden kann.

Neben den Betrachtungen für den Regelbetrieb wurden auch Untersuchungen zu Szenarien unter erschwerten Bedingungen durchgeführt. Beispielsweise kann ein erhöhter Hilfsbetriebeenergiebedarf (z.B. Klimaanlage) aufgrund von Temperatureinflüssen zu erhöhter Energieentnahme aus 
KBS: 227,1 von Kamenz (Sachs) nach Dresden Hbf tief

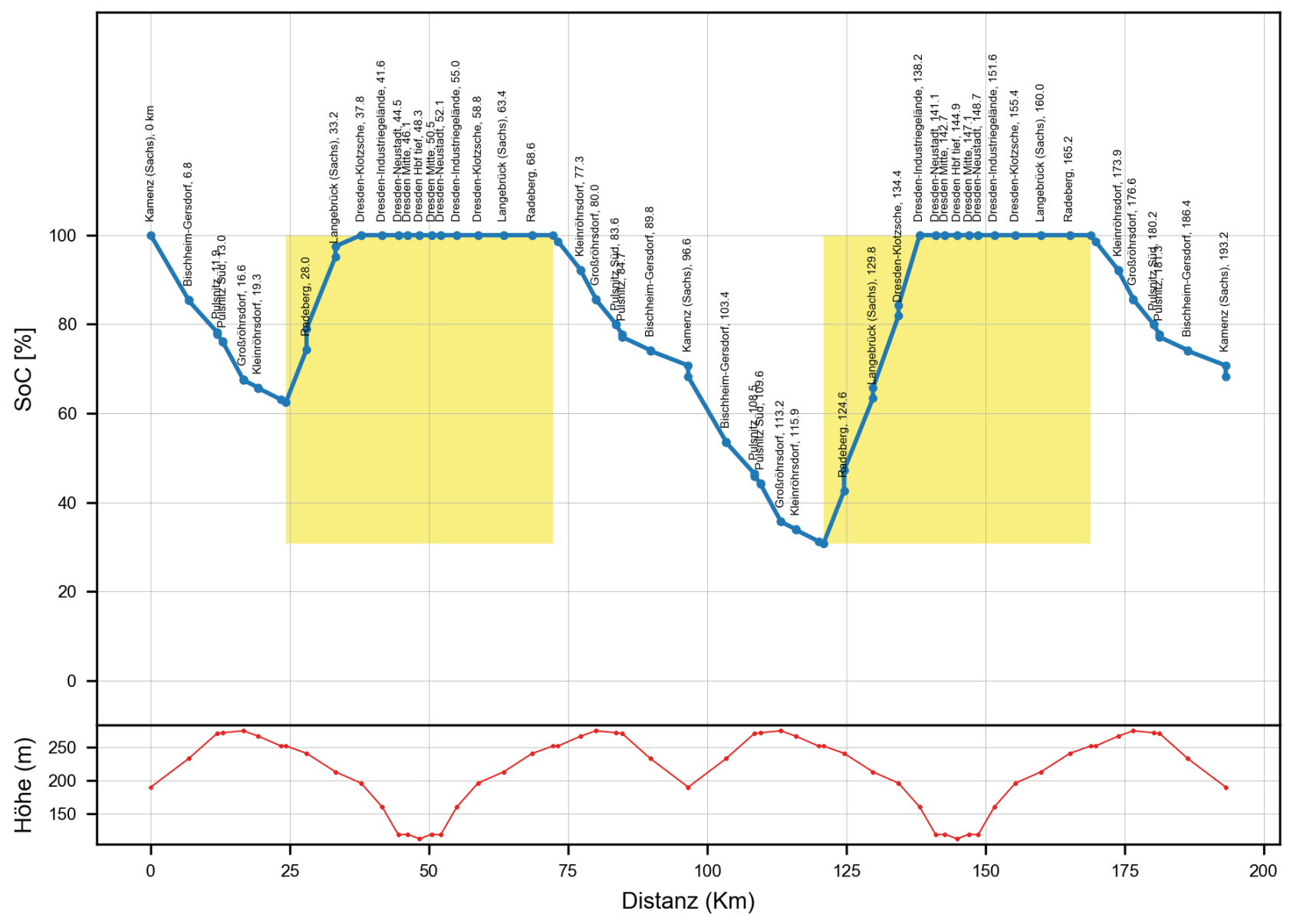

Quelle: MPM

der Batterie führen. Die Abbildung 7 zeigt einen solchen Verlauf, der aber nur an wenigen Tagen im Jahr zu erwarten ist. Eine detaillierte Untersuchung zu den Verläufen des Energieverbrauchs in Abhängigkeit der Umgebungstemperaturen steht noch aus.

\section{Betriebliche Randbedingungen}

Aufgrund der notwendigen Nachladung von Batterietriebzügen kommt der Wendezeit an den jeweiligen Endbahnhöfen einer Linie eine besondere Bedeutung zu. Wendezeiten können als Puffer dienen, durch den aufgelaufene Verspätungen wieder aufgeholt werden können. Bisher besteht der bestimmende Faktor darin, dass das Fahrzeug die Fahrtrichtung und der Triebfahrzeugführer den Führerstand wechseln muss. Dies konstituiert die sogenannte Mindestwendezeit. Im Batteriezugbetrieb kommt nun an Endbahnhöfen, die eine Lademöglichkeit bieten, der Faktor der Ladezeit hinzu. Es muss gewährleistet sein, dass die Ladezeit ausreicht, um den Zug sicher durch den nächsten oberleitungsfreien Ab- schnitt zu bringen. Hierfür ist die Festlegung einer Mindestenergiemenge in der Batterie sinnvoll, vor deren Erreichen der Zug nicht abfahren darf. Aufgrund der unterschiedlichen Charakteristika der einzelnen SPNV-Linien muss dieser Wert in jedem Einzelfall neu festgelegt werden. Sofern die Wendezeiten des bisherigen Fahrplans für eine Ladung ausreichen, müssen keine weiteren Schritte ergriffen werden, sobald sich aber der Fahrplan ändern soll, muss der Aspekt der ausreichenden Ladezeiten geprüft werden. Dies kann auch dazu führen, dass sich bisherige Wendezeiten verlängern und beispielsweise eine überschlagene Wende notwendig ist, was für gewöhnlich eine Erhöhung des Fahrzeugbedarfes zur Folge hat.

Daraus folgt, dass eine Positionierung von Ladeinfrastruktur an Linienendpunkten sinnvoll ist. Die Standzeit ist dort in den meisten Fällen höher als an Kreuzungspunkten im Verlauf der Linien, wodurch eine größere Energiemenge in das Fahrzeug gebracht werden kann. Im Vergleich zu (Teil-) Elektrifizierungen sind Ladestationen für eine Aufladung im Stand perspektivisch einfacher zu errichten als Oberleitungsanlagen, unter denen auch gefahren werden soll. Aller- 
Abbildung 7: Simulation der RB 34 mit Elektrifizierung Dresden-Klotzsche - Arnsdorf (erhöhter Hilfsbetriebeenergiebedarf)

KBS: 227,1 von Kamenz (Sachs) nach Dresden Hbf tief

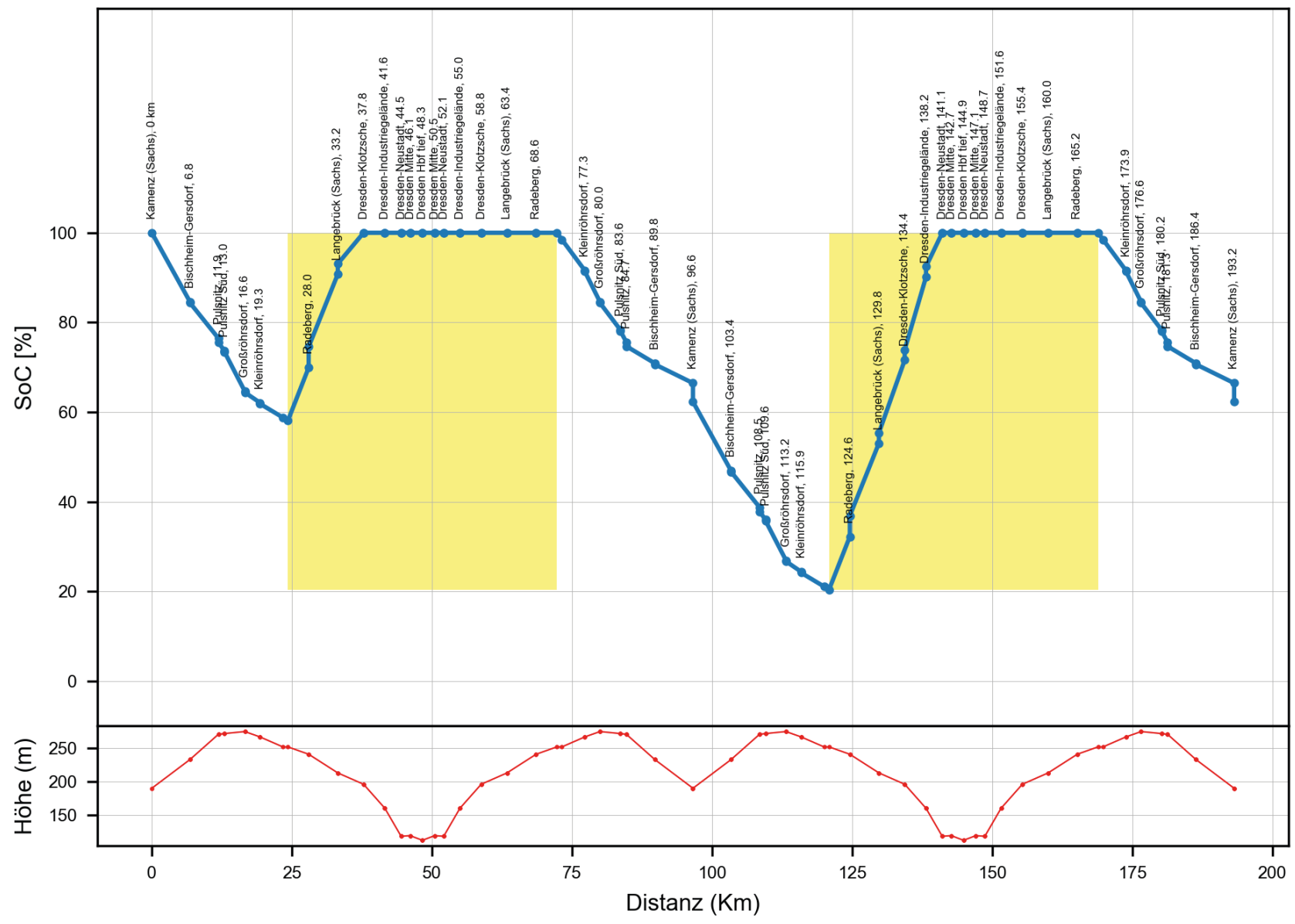

Quelle: MPM

dings befinden sich die Endpunkte meist weit weg vom traditionellen Bahnstromnetz, sodass eine Versorgung aus dem Landesnetz der Energieversorger notwendig ist, die im Einzelfall zu prüfen ist. In anderen Fällen kann es vorkommen, dass eine Veränderung der Linienzuschnitte im betrachteten Netz die Errichtung von Ladeinfrastruktur obsolet macht. Da eine Mischung von elektrifizierten und nichtelektrifizierten Abschnitten der Charakteristik eines Batteriefahrzeuges am meisten entspricht, ist es beispielsweise bei Vorliegen der entsprechenden Voraussetzungen möglich, ein Liniennetz auf diesen Aspekt hin anzupassen. Diese Möglichkeit war im VVO-Netz nicht gegeben.

Aus den hohen Beschleunigungswerten von Elektrotriebzügen ergeben sich in vielen Fällen Fahrzeitgewinne gegenüber bisher eingesetzten Dieselfahrzeugen. Ob und in welcher Höhe diese auftreten, ist sowohl vom bisherigen Fahrplan als auch von den fahrdynamischen Eigenschaften der bisher eingesetzten Fahrzeuge abhängig. Auch die Nutzung dieser Fahrzeitgewinne muss im Einzelfall betrachtet werden, da die Möglichkeiten stark davon abhängig sind, ob die Fahrzeitgewinne auf einer ein- oder zweigleisigen Strecke entstehen und im Weiteren davon, wie der aktuelle Fahrplan aussieht. Zum einen können die Fahrzeitgewinne als Puffer für Verspätungsabbau genutzt werden, was den häufigeren Anwendungsfall darstellt, insbesondere auf kürzeren Linien. Dies dient auf jeden Fall der Erhöhung der betrieblichen Stabilität. Je nach Konstellation kann aber die Fahrzeitverkürzung so groß sein bzw. so geschickt liegen, dass tatsächlich eine Fahrzeugeinsparung möglich ist, wie es im untersuchten Netz auch vorgekommen ist. Weiterhin besteht das Potential für Fahrplananpassungen, was im Rahmen der VVO-Studie allerdings nicht untersucht wurde. Aufgrund der vielen Abhängigkeiten innerhalb eines Liniennetzes (wie beispielsweise eingleisige Abschnitte, höhengleiche Kreuzungen, Trassenbelegungen in vielbefahrenen Abschnitten) sind derartige Anpassungen sehr aufwändig. Es ist aber für einen erfolgreichen Betrieb mit Batterietriebzügen notwendig, dass sowohl ihre Potentiale als auch ihre Restriktionen in der Fahr- und Umlaufplanung berücksichtigt werden. Das betrifft auch Aspekte wie den Wegfall von Tankfahrten im Umlaufplan oder die Prüfung, ob Reinigung und Entsorgen unter Oberleitung oder im Batteriemodus durchgeführt werden. 
Das Thema Reichweite von Batteriefahrzeugen wird bisher sehr stark von fixen Kilometerwerten bestimmt, die verhältnismäßig einfach das Leistungsvermögen des Fahrzeugs veranschaulichen sollen. Es gibt allerdings bisher keine definitorische Einordnung, unter welchen Bedingungen diese Werte gelten. Grundlegende Rahmenbedingungen wie Wettereinflüsse, Besetzungsgrad des Fahrzeugs sowie Infrastrukturparameter werden bei Reichweitenangaben in der Bahnbranche nicht berücksichtigt. Hier wäre es ratsam, zukünftig einen Normzyklus zur besseren Vergleichbarkeit der Fahrzeuge zu nutzen. Es muss aber klar sein, dass zu einer validen Aussage über die Eignung einzelner Liniennetze auf jeden Fall die dort geltenden Randbedingungen zu betrachten sind.

Besondere Bedeutung kommt aufgrund der deutlich begrenzten Reichweite von Batterietriebzügen der Betrachtung von Störungseinflüssen auf das Fahrzeug zu. Diese können völlig unterschiedlicher Natur sein. Von Störungen der Infrastruktur über Störungen am Fahrzeug zu betrieblichen Verzögerungen müssen alle Möglichkeiten zumindest geprüft werden. Letztlich lassen sich alle Störungseinflüsse auf das Grundproblem reduzieren, dass der nächste elektrifizierte Bahnhof / Haltpunkt oder Streckenabschnitt erreicht werden muss, ohne dass das Fahrzeug tatsächlich ohne Traktionsenergie liegenbleibt. Hierzu kommen in der Betrachtung zwei Pufferzonen zum Tragen. Zum einen ist es sinnvoll, im Bereich der dauerhaft nutzbaren Batteriekapazität vorzusehen, dass diese nicht ständig bis zu $0 \%$ entladen wird. Zum zweiten gibt es den bereits oben erwähnten Tiefentladebereich, der vom Fahrzeughersteller bzw. durch die verwendete Batterietechnologie vorgegeben wird, dessen Nutzung aber mit der Verkürzung der Batterielebensdauer einhergehen kann. Die Nutzung dieses Tiefentladebereiches ist also unter Gesichtspunkten von Wirtschaftlichkeit und Umweltbilanz auf ein Minimum zu reduzieren.

Um das oben illustrierte Beispiel der RB 34 noch einmal aufzugreifen: Es wurde festgestellt, dass unter den gegebenen Rahmenbedingungen zusätzliche Infrastruktur notwendig ist, beispielsweise eine Ladestation in Kamenz. Für den Fall, dass die dortige Ladestation ausfällt und somit kurzzeitig nicht zur Verfügung steht, wäre ein ausreichender Puffer betrieblich sinnvoll, um in Dresden-Klotzsche die Oberleitung wieder zu erreichen. Ansonsten müsste der Zugbetrieb mit Batteriefahrzeugen dort temporär eingestellt werden. Die Simulation des Status quo (Abbildung 4) zeigt, dass eine Befahrung der Linie auch bei ausgefallener Ladestation möglich ist, zumindest unter Nutzung des Tiefentladebereiches der Batterie. Für den Störungsfall ist auch eine Reduzierung der Versorgung der Hilfsbetriebe im Zug möglich, um ein Liegenbleiben zu vermeiden.

Es stellt sich aber die Frage, inwieweit es wirtschaftlich sinnvoll ist, jede Störung beliebiger Größe durch eine Vergrößerung der Batteriekapazität abzufangen. Gewisse Ereignisse würden als Restrisiko bleiben und müssten auf andere Weise behandelt werden. Wenn man den Vergleich zum konventionellen elektrischen Bahnbetrieb unter Oberleitung heranzie- hen möchte: Sollte die Oberleitung stromlos oder beschädigt sein, ist in den jeweiligen Abschnitten kein elektrischer Zugbetrieb mehr möglich. Eine technische Rückfallebene gibt es hierfür nicht. Nun ist die Ausfallwahrscheinlichkeit von Bahnstromanlagen sehr gering [14], allerdings liegen für abgesetzte Ladeinfrastruktur, die aus dem Landesnetz versorgt wird, noch keine Zahlen vor. Eine Einschätzung, wie oft der jeweilige Fall vorkommt, ist damit noch recht schwierig.

Grundsätzlich ist in der Detailbetrachtung einzelner Linien die Berücksichtigung von Störeinflüssen in nahezu beliebigem Detaillierungsgrad möglich. Gerade Einflüsse im Bereich Infrastruktur sind je nach Betrachtungsraum sehr verschieden in ihrer Ausprägung und der Wahrscheinlichkeit des Auftretens. Exemplarisch seien hier nur Behinderungen durch gestörte Bahnübergänge oder ins Gleis gefallene Bäume genannt, die lokal sehr unterschiedlich auftreten.

\section{Zusammenfassung}

Das VVO-Netz bietet aufgrund seiner zum Teil topologisch sehr anspruchsvollen Linienführung einen interessanten Untersuchungsraum für Betrachtungen zum Batteriezugbetrieb. Es kann davon ausgegangen werden, dass viele Aussagen, die für das untersuchte Netz gelten, auch in anderen Netzen ähnlich gültig und zum Teil sogar verallgemeinerbar sind.

Aus betrieblicher Sicht ist besonders relevant, dass die Fahr- und Wendezeiten des bisherigen Fahrplans mit den Batteriefahrzeugen eingehalten werden können, sofern dies Maßgabe der Aufgabenstellung ist. Dies war in der bearbeiteten Studie der Fall und wurde entsprechend eingehalten. Wenn es größere Spielräume für Änderungen am Fahrplanoder Linienkonzept gibt, sollten diese genutzt werden, wo dies die Eignung des Netzes für Batterietriebzüge verbessert. Potentiale für kürzere Fahrzeiten können beispielsweise für die Erhöhung der Betriebsstabilität genutzt werden. Genügend Puffer für eventuelle Störeinflüsse, zum Beispiel Verspätungen oder Störungen an der Infrastruktur, müssen vorhanden sein. Gerade die Überlegungen zum Thema Störungsbetrieb müssen in jeder Untersuchung dieser Art eine wichtige Rolle spielen.

Hinsichtlich der Positionierung von Ladestationen hat sich grundsätzlich ergeben, dass Endstellen von Linien aus betrieblicher Sicht besonders prädestiniert sind, da die Wendezeit als Ladezeit genutzt werden kann. Erst wenn dies alleine nicht ausreicht, sollte über weitere Möglichkeiten wie Teilelektrifizierungen nachgedacht werden. Ebenso sollte in jedem Einzelfall untersucht werden, ob die Errichtung von Infrastruktur nicht durch planerische oder betriebliche Maßnahmen vermieden werden kann.

Die Simulationssoftware eFlips erwies sich als gut geeignet und konnte durch Erkenntnisse aus der Bearbeitung der Studie noch in einzelnen Aspekten verbessert werden. Entsprechende Rückfragen seitens des VVO, die im Laufe der Studie auftraten, konnten aufgenommen und in das Modell 
integriert werden, sodass eine gute praktische Anwendbarkeit der Ansätze gegeben ist.

Alle in diesem Artikel aufgeworfenen Fragestellungen wurden am Beispiel des VVO-Dieselnetzes untersucht und beantwortet, soweit dies zu Erfüllung des Studienzweckes notwendig war. Darüber hinaus sind aber sicherlich diese Fragen auch in ihrer Gesamtheit interessant und eine Behandlung im größeren Kontext des möglichen Einsatzes von Fahrzeugen mit alternativen Antriebssystemen im gesamten deutschen SPNV daher geboten. Gerade im Bereich der Störungseinflüsse sind größere systematische Untersuchungen des Einflusses auf den Betrieb eines Batteriezugnetzes noch im Aufbau und der Forschungsbedarf entsprechend gegeben.

\section{Das Verbundprojekt BEMU}

Das Fachgebiet Bahnbetrieb und Infrastruktur übernimmt gemeinsam mit dem Fachgebiet Methoden der Produktentwicklung und Mechatronik an der TU Berlin die wissenschaftliche Begleitung der Entwicklung eines Batterietriebzugs durch Bombardier Transportation. Das vom Bundesministerium für Verkehr geförderte und von der NOW (Nationale Organisation Wasserstoff- und Brennstoffzellentechnologie) koordinierte Projekt umfasst die Entwicklung und Bau eines TALENT 3 Triebzuges, welcher mithilfe eines zusätzlichen Batteriesystems oberleitungsfreie Abschnitte überbrücken oder ganze nichtelektrifizierte Streckenabschnitte befahren kann. Zur InnoTrans 2018 im September in Berlin ist das Fahrzeug fahrtauglich öffentlich präsentiert worden. Es soll ein 12-monatiger Fahrgastbetrieb in Zusammenarbeit mit der DB RAB und der NVBW (Nahverkehrsgesellschaft Baden-Württemberg) in Baden-Württemberg erfolgen.

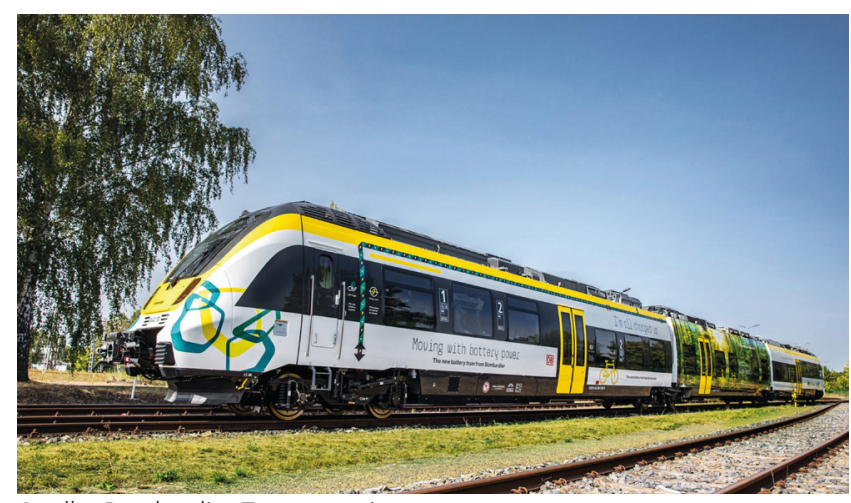

Quelle: Bombardier Transportation

\section{Literaturangaben}

[1] M. Hecht, „Schienenverkehr neu organisieren“, Tagesspiegel, 10.06.2018.

[2] Allianz pro Schiene, „Bund noch ohne Plan für Streckenelektrifizierung", 18.12.2018. [Online]. Available: https:// www.allianz-pro-schiene.de/presse/pressemitteilungen/ bund-noch-ohne-plan-fuer-streckenelektrifizierung/. [Zugriff am 15.08.2019].

[3] M. Hecht und C. Culemann, „Klimaschutz als Chance für die Bahn“, Deine Bahn, Bd. 12/2018, pp. 14-17, 2018.

[4] CDU/CSU, SPD, „Koalitionsvertrag zwischen CDU, CSU und SPD“, 12.03.2018. [Online]. [Zugriff am 15.08.2019].

[5] S. von Mach, "TALENT 3 Batterietriebzug BEMU“, 21.02.2017. [Online]. Available: https://www.now-gmbh. de/content/1-aktuelles/1-presse/20170221-fachkonferenz-elektromobilitaet-vor-ort/1_14_vonmach_bemu.pdf. [Zugriff am 15.08.2019].

[6] K. Hasebe, N. Shiraki und H. Takiguchi, „Special FeatureJR East EV-E301 Catenary and Battery-Powered Hybrid Railcar", Japan Railway \& Transport Review No. 65, pp. 36-43, 2015.

[7] S. Schrader, „Heimliches Debüt für Batteriezug von Stadler", Eisenbahn Revue International, Nr. 11, p. 578, 2018.

[8] bei, „ÖBB-Desiro mit zusätzlichem Batterieantrieb vorgestellt", Eisenbahn Revue International, Nr. 11, p. 576, 2018.

[9] S. Schrader, „Bombardier stellt Talent 3 mit Batterie-Zusatzantrieb vor", Eisenbahn Revue International, Nr. 11, p. $578,2018$.

[10] „Bahnverkehr wird umweltfreundlich“, 2019. [Online]. Available: https://www.schleswig-holstein.de/DE/Landesregierung/VII/_startseite/Artikel2019/II/190619_VergabeXMU.html. [Zugriff am 15.08.2019].

[11] Fraunhofer-Institut für System- und Innovationsforschung, „Energiespeicher-Roadmap (Update 2017)“, Karlsruhe, 2017.

[12] D. Jefferies, „eFLIPS: Electric Fleet and Infrastructure Planning/Simulation“, 2019. [Online]. Available: https:// www.mpm.tu-berlin.de/menue/forschung/projekte/eflips/. [Zugriff am 15.08.2019].

[13] I. Kramer und T. Berger, „Frühestens 2029 pendeln Elektrozüge zwischen Dresden und Görlitz", Sächsische Zeitung, p. 8, 08.11.2018.

[14] Sächsische Staatsregierung, „Leuchtturmprojekte für Braunkohleregionen vorgestellt", 29.04.2019. [Online]. Available: https://www.staatsregierung.sachsen.de/aktuelle-entwicklungen-zum-strukturwandel-6064.html. [Zugriff 12.09.2019].

[15] F. Kießling und R. S. A. Puschmann, Fahrleitungen elektrischer Bahnen, Berlin: Publicis Publishing, 2014, p. 1171. 